\title{
Optometric Clinical Practice
}

Volume 2 | Issue 1

\section{Silent Sinus Syndrome: An Uncommon Cause of Diplopia}

Tyler V. Mullen OD

Department of Veterans Affairs, tvmullen@gmail.com

Ruth Hyatt OD, FAAO, Diplomate

Department of Veterans Affairs, ruth.hyatt@va.gov

Darcy Eberle OD

Department of Veterans Affairs, Darcy.Eberle@va.gov

Follow this and additional works at: https://athenaeum.uiw.edu/optometric_clinical_practice

Part of the Optometry Commons

The Athenaeum provides a publication platform for fully open access journals, which means that all articles are available on the Internet to all users immediately upon publication. However, the opinions and sentiments expressed by the authors of articles published in our journal does not necessarily indicate the endorsement or reflect the views of the University of the Incarnate Word and its employees. The authors are solely responsible for the content of their work. Please address questions to athenaeum@uiwtx.edu.

\section{Recommended Citation}

Mullen TV, Hyatt R, Eberle D. Silent Sinus Syndrome: An Uncommon Cause of Diplopia. Optometric Clinical Practice. 2020; 2(1). doi: 10.37685/uiwlibraries.2575-7717.2.1.1005. https://doi.org/10.37685/ uiwlibraries.2575-7717.2.1.1005

This Case Study is brought to you for free and open access by The Athenaeum. It has been accepted for inclusion in Optometric Clinical Practice by an authorized editor of The Athenaeum. For more information, please contact athenaeum@uiwtx.edu. 


\section{Silent Sinus Syndrome: An Uncommon Cause of Diplopia}

Abstract

Background: Silent sinus syndrome is a rare condition with multiple ophthalmic findings of significance. Chronic, subclinical maxillary sinus inflammation alters the pressure gradient, leading to eventual maxillary sinus collapse, orbital floor depression, enophthalmos, and diplopia.

Case Report: Functional endoscopic sinus surgery may be indicated to correct diplopia and/or cosmesis.

Conclusion: Patients unbothered by facial asymmetry may be managed with prism in eyeglasses.

\section{Keywords}

diplopia, sinus, enophthalmos, hypoglobus

Creative Commons License

(c) (i) (8)

This work is licensed under a Creative Commons Attribution-Noncommercial 4.0 License 


\section{INTRODUCTION}

Silent sinus syndrome (SSS) is a rare condition with multiple ophthalmic findings of significance. Chronic, subclinical inflammation and subsequent altered pressure gradient of the maxillary sinus leads to its eventual collapse. The resulting depression of the orbital floor often leads to hypoglobus and enophthalmos. Radiologic imaging is critical for diagnosis. ${ }^{1}$ It should be noted that 'silent' refers to absence of sinusitis symptoms rather than ocular symptoms. ${ }^{2}$

\section{CASE REPORT}

A 67-year-old white male presented with chief complaint of painless, constant vertical diplopia in primary gaze that had been present for six to eight months. He had no history of trauma and did not complain of headache, fever, malaise, jaw claudication, or scalp tenderness. He underwent cataract surgery with posterior chamber intraocular lens implantation in both eyes five years prior. He was prescribed atorvastatin calcium $20 \mathrm{mg}$ daily for cholesterol, 8 units aspart insulin three times daily and $500 \mathrm{mg}$ metformin $\mathrm{HCl}$ twice daily for type 2 diabetes, levothyroxine sodium $0.05 \mathrm{mg}$ daily for hypothyroidism, sildenafil citrate $100 \mathrm{mg}$ daily for erectile dysfunction, and warfarin sodium $5 \mathrm{mg}$ three times daily for history of venous thrombosis.

Best corrected vision measured 20/20 in the right and left eyes. Pupils were equal, round, and reactive to light without afferent defect. Confrontation visual fields were full. Extraocular motility was intact. He demonstrated 12 prism diopters of left hypertropia and 2 prism diopters of exophoria at distance. There was $4 \mathrm{~mm}$ of right hypoglobus (Figure 1) without ptosis. Exophthalmometry was $21 \mathrm{~mm}$ in both eyes.

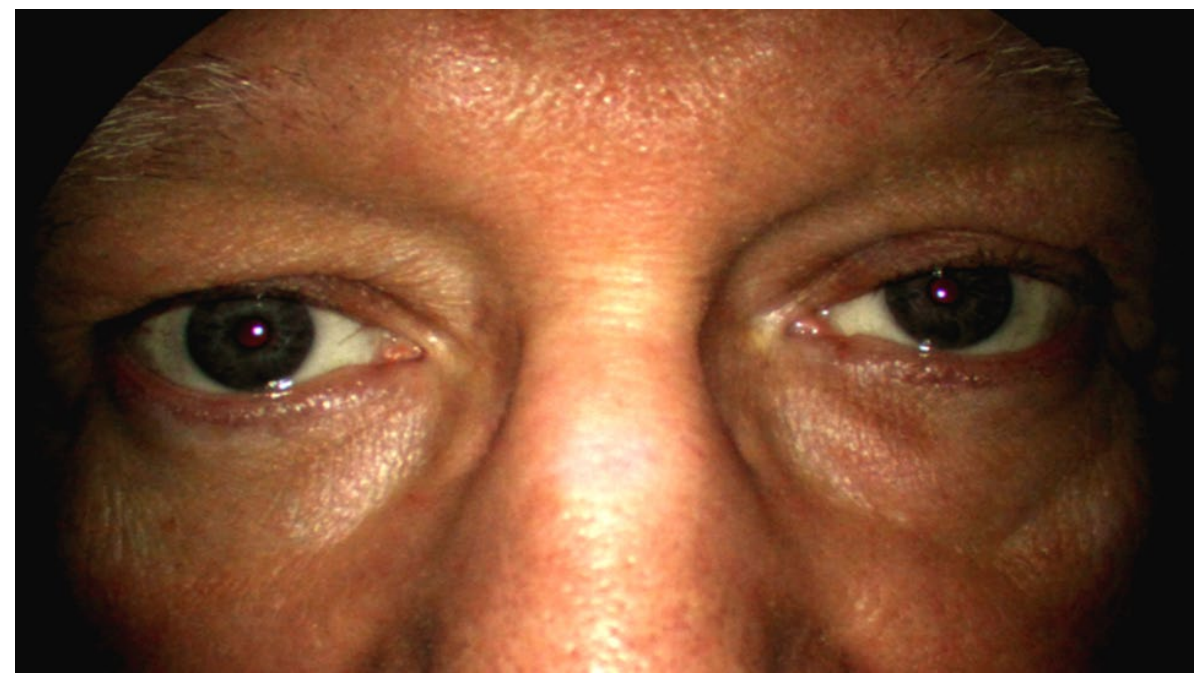

Figure 1: Right hypoglobus measuring $4 \mathrm{~mm}$

Intraocular pressure measured $16 \mathrm{mmHg}$ in the right and left eyes. Both eyes had mild corneal gutatta, clear posterior chamber intraocular lenses, 0.25 cup-to-disc ratio without 
edema, flat and intact maculae and peripheral retinas without choroidal folds. Optical coherence tomography showed normal foveal contour without sub- or intra-retinal fluid.

Vasculopathic etiology of diplopia was ruled out as resolution would be expected within three months ${ }^{3}$ and this patient's symptoms were present for at least six months. Statins have been associated with the precipitation of myasthenia gravis, ${ }^{4,5}$ however there was no fluctuation in symptoms, particularly at the end of the day., Inflammatory etiology (such as thyroid eye disease or giant cell arteritis) was considered, however hypoglobus is unexpected in both conditions; headache, fever, malaise, jaw claudication, and scalp tenderness were absent. ${ }^{3}$ The patient was being treated medically for subclinical hypothyroidism for 2 years and had normal TSH levels 2 months prior. Pupil symmetry and lack of ptosis ruled out cranial nerve III palsy and Horner's syndrome. ${ }^{3,5}$ He had no history of trauma.

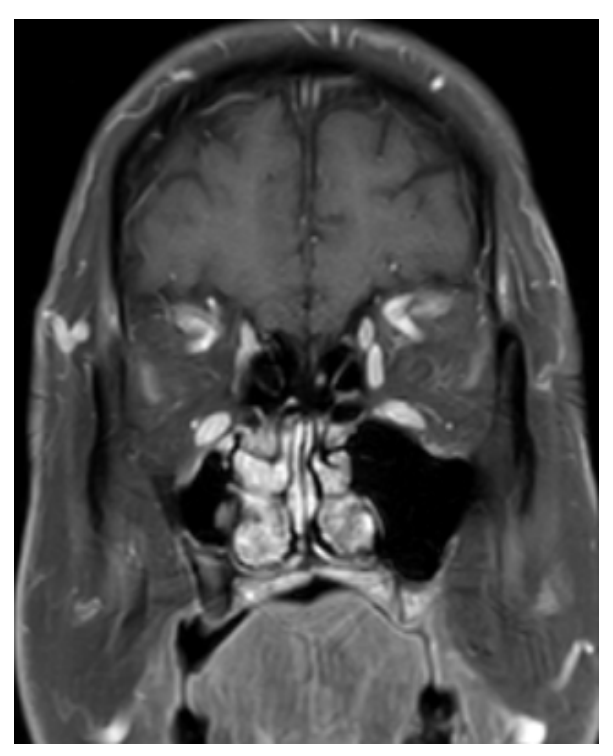

Figure 2 (left):

Before onset of diplopia, MRI with contrast revealed diminutive right maxillary sinus and chronic right maxillary sinus disease.

Figure 3 (right): MRI with contrast through right orbit and maxillary sinus.

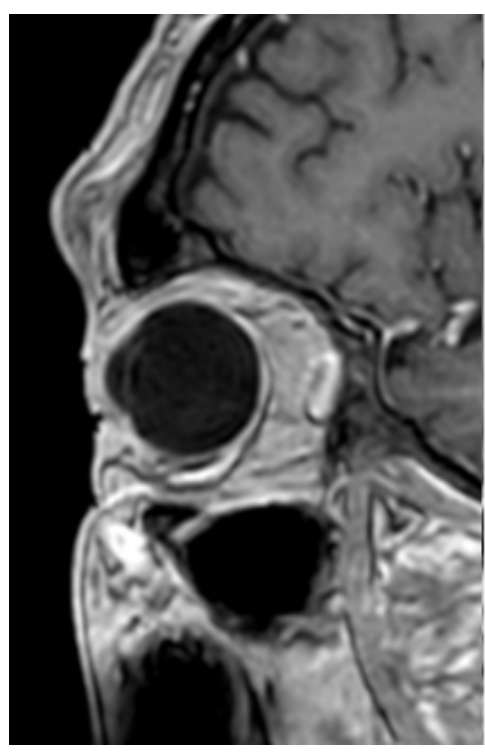

Several months prior to the onset of diplopia, the patient's primary care provider ordered contrasted magnetic resonance imaging (MRI) of the brain because of ataxia. An incidental finding of right maxillary sinus disease (Figures 2 and 3) without hypoglobus was revealed.

New diplopia with hypoglobus prompted ordering of comparison contrasted MRI of the face and orbits, which demonstrated interval change with decreased right maxillary sinus size, depression of the right orbital floor and confirmed lower globe position, suggesting silent sinus syndrome (Figures 4 and 5).

The patient's diplopia was relieved with eyeglasses having 7 diopters base up prism in the right eye. He was unbothered by facial asymmetry and declined consultation with oculoplastics for surgical correction. 

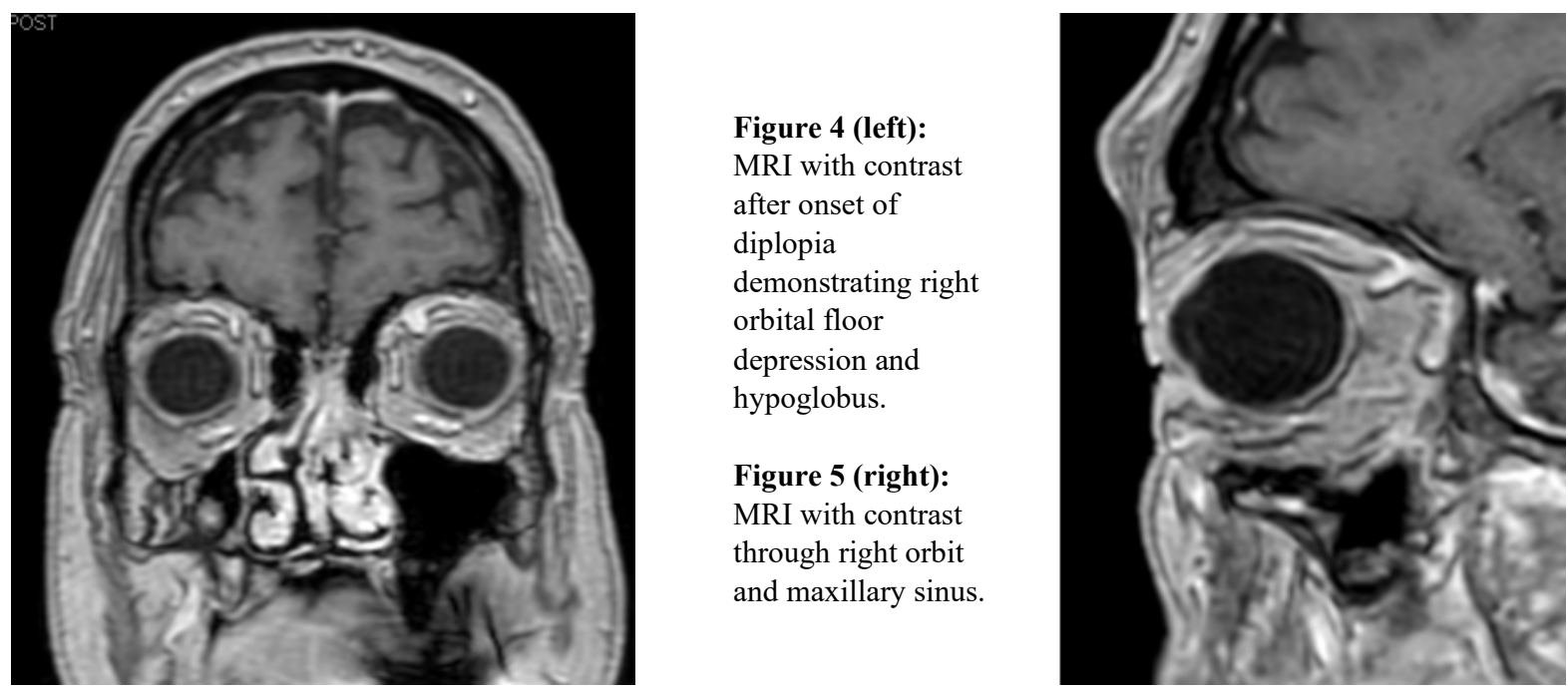

\section{DISCUSSION}

Silent sinus syndrome was first described by Montgomery in 1964 but not named until 1994 by Soparkar and colleagues. ${ }^{1,6,7}$ It consists of asymptomatic maxillary sinus hypoplasia that leads to abnormalities of the ipsilateral orbital floor. These abnormalities usually manifest as bone resorption and remodeling which leads to spontaneous enophthalmos and/or hypoglobus. ${ }^{6,8}$

Chronic maxillary atelectasis (CMA) represents a spectrum of maxillary sinus disease that in its late stages can manifest as SSS. In CMA and SSS, symptoms are inversely related to anatomical changes; that is, those who are asymptomatic have greater sinus changes apparent on radiologic imaging. ${ }^{9}$ Symptoms of sinus disease may or may not be present in CMA, and patients are more likely to be asymptomatic when more advanced disease (such as SSS) is present. ${ }^{8,9}$ Soparkar's diagnostic criteria for SSS include modification of facial appearance, hypoglobus and/or enophthalmos, decreased maxillary sinus volume on radiologic imaging, and the lack of sinus-related symptoms. ${ }^{6}$

Although SSS is a late-stage manifestation of CMA, no extensive studies on the incidence of SSS exist, possibly due to the rare nature of the condition. Depending on the literature source, there is wide variability in diagnostic nomenclature between CMA and SSS, with the term "CMA" more commonly used in otolaryngology literature and "SSS" favored in ophthalmology literature. ${ }^{9}$ For example, Numa and colleagues ${ }^{1}$ reviewed all 84 reported cases of SSS in 2005. Three years later, Brandt and colleagues ${ }^{9}$ determined that 32 out of the 62 journal articles they identified met their review criteria; of those, only 55 cases of SSS met what they defined as a complete data set and of those, only 28 cases met the diagnostic criteria for SSS. Using this methodology, they determined that $85 \%$ of SSS met diagnostic criteria for CMA and proposed abandoning the term SSS in favor of CMA, as both seem to be on a spectrum of the same clinical entity, with SSS being a late-stage manifestation of CMA. 
The development of SSS is brought on by a buildup of negative pressure within the maxillary sinus. The ostiomeatal complex is the opening between the maxillary sinus and the surrounding tissues. A flap-valve effect of the uncinate process incites a blockage of the ostiomeatal complex. Gas resorption into the capillaries leads to negative pressure build up and an accumulation of inflammatory secretions within the maxillary sinus. Inward bowing of the sinus wall leads to bone resorption and remodeling of the orbital floor. This negative pressure and inward bowing of the sinus wall can lead to depression of the orbital floor, the weakest wall of the maxillary sinus. The resultant change in orbital anatomy causes hypoglobus and/or enophthalmos. ${ }^{1,9,10}$

Patients with SSS may present with one or more ophthalmic findings of significance. Findings most likely to be found on clinical examination include enophthalmos (98$100 \%)^{1,9}$ and hypoglobus (44-53\%), ${ }^{9}$ measuring on average $2.96 \mathrm{~mm}$ and $2.78 \mathrm{~mm}$ respectively. ${ }^{11}$ Although extraocular motility remains normal in a majority of cases, ${ }^{9,12}$ there are reported cases of altered superior and inferior oblique muscle action due to a relative dislocation between the extraocular muscle insertion points at the globe and the muscle origins on the orbit. ${ }^{1}$ Other findings include diplopia $(28-65 \%)^{1,9}$ and history of sinus disease $(56 \%))^{7}$ Measurements of lid abnormality can also aid in evaluation and diagnosis. Enophthalmos can lead to a decreased palpebral fissure. Hypoglobus can lead to an increased margin crease distance and exposure-related dryness. Leading chief complaint is facial asymmetry $(69 \%)$ followed by diplopia $(65 \%){ }^{9}$. Mean symptom duration is 6.5 months. ${ }^{1}$ Right- and left-sided disease incidence is equal and there is no gender predilection. ${ }^{9}$ Visual acuity usually remains intact. ${ }^{9}$

Radiologic imaging is essential for diagnosis, and CT and MRI are both acceptable imaging modalities. ${ }^{12}$ In a 2008 review of cases by Brandt and Wright, maxillary sinus contraction was seen in $100 \%$ of patients. ${ }^{9}$ The next most common radiologic findings include: orbital floor depression $(87 \%)$, opacification of the sinus $(84 \%)$, other sinus disease $(61 \%)$, and inward bowing of maxillary walls $(47 \%) .{ }^{9}$ Given that there is a lack of sinus symptoms in patients diagnosed with SSS, surgical intervention may not always be indicated. Prism correction of diplopia may be sufficient in patients who are unconcerned with cosmesis. It should be noted that vertical strabismus measurement in prism diopters is independent of hypoglobus measurement in millimeters. Cover test with prism neutralization or other binocular vision tests should be done in addition to measuring hypoglobus using the corneal light reflex. If the Hirschberg reflex is used to aid in determining prescribed prism, the distance between the reflex in strabismic posture and the visual axis should be used; this is separate from the measurement of hypoglobus using the corneal light reflex. In patients motivated to restore normal facial appearance or improve diplopia without optical correction, surgical intervention is necessary.

Surgical approaches are separated into sinonasal and orbital. Functional endoscopic sinus surgery (FESS) is the gold standard sinonasal approach. ${ }^{9}$ The main objectives of FESS are to restore drainage of the maxillary sinus (which will prevent future sinus disease), and decrease enophthalmos and/or hypoglobus (indirectly restoring orbital anatomy). ${ }^{13}$ FESS begins with a septoplasty to allow access to the maxillary sinus. An uncinectomy may be performed to re-establish drainage through the osteomeatal complex. An antrostomy enlarges the maxillary sinus ostium. These steps alone may re-establish sinus ventilation 
and allow for the orbital floor to rise to its normal position, ${ }^{9}$ effectively avoiding orbital surgery. It is hypothesized that surgical correction of the maxillary sinus can indirectly improve hypoglobus and enophthalmos due to poorly understood alterations that occur in the maxillary sinus after surgery. ${ }^{11}$ However, some surgeons prefer to complete orbital floor reconstruction immediately following FESS. Concomitant orbital floor repair avoids a second operation and anesthesia administration yet is more invasive, increases surgical time, and leads to an identical outcome in many patients. ${ }^{1,2,11,13}$

\section{CONCLUSION}

Although infrequently encountered, silent sinus syndrome represents a condition that is likely to first present to an eye care provider. This case report demonstrates the importance of understanding differential diagnoses of diplopia. The natural history of silent sinus syndrome is evidenced by interval change demonstrated by MRI. The eye care provider should aim for a team approach with radiologic, otolaryngology, and oculoplastic services for both diagnosis and management of this unusual condition.

\section{REFERENCES}

1. Numa WA, Desai U, Gold DR, Heher KL, Annino D. Silent sinus syndrome: a case presentation and comprehensive review of all 84 reported cases. Ann Otol Rhinol Laryngol. 2005;114(9):688-94. doi: 10.1177/000348940511400906

2. Pula JH, Mehta M. Silent sinus syndrome. Curr Opin Ophthalmol. 2014;25(6):480-4. doi: 10.1097/ICU.0000000000000106

3. Williamson, J. Seeing double: the urgent and chronic causes of diplopia. Rev Optom. March 15, 2015:63-70.

4. Gale J, Danesh-Meyer H. Statins can induce myasthenia gravis. J Clin Neurosci. 2014;21:195-7. doi: 10.1016/j.jocn.2013.11.009

5. Malloy K. Neuro-ophthalmic disease basics: evaluating the efferent visual system. Rev Optom. February 15, 2015: 74-82.

6. Soparkar CNS, Patrinely JR, Cuaycong MJ, et al. The silent sinus syndrome: a cause of spontaneous enophthalmos. Ophthalmology. 1994;101(4):772-8.

doi: 10.1016/s0161-6420(94)31267-x

7. Montgomery WW. Mucocele of the maxillary sinus causing enophthalmos. Eye Ear Nose Throat Mon. 1964;43:41-4.

8. Ende K, Mah L, Kass ES. Progression of late-stage chronic maxillary atelectasis. Ann Otol Rhinol Laryngol. 2002;111(8):759-62. doi: 10.1177/000348940211100819 
9. Brandt MG, Wright ED. The silent sinus syndrome is a form of chronic maxillary atelectasis: a systematic review of all reported cases. Am J Rhinol. 2008;22(1):68-73. doi: $\underline{10.2500 / \text { ajr.2008.22.3118 }}$

10. Elkhatib A, McMullen K, Hachem RA, Carrau RL, Mastros N. Rapidly progressive maxillary atelectasis. J Craniofac Surg. 2017;28(5):e451-3. doi:

10.1097/SCS.0000000000002890

11. Babar-Craig H, Kayhanian H, De Silva DJ, Rose GE, Lund VJ. Spontaneous silent sinus syndrome (Imploding antrum syndrome): case series of 16 patients. Rhinology. 2011;49(3):315-7. doi: 10.4193/Rhino10.103

12. Gill H, Silkiss R. Diagnosis and management of silent sinus syndrome. Eyenet. July/August 2011:37-38. https://www.aao.org/eyenet/article/diagnosis-managementof-silent-sinus-syndrome

13. Trombitas V, Nagy Alina, Vlad D, Ilea A, Albu S. Silent sinus syndrome - case presentation. Romanian J Rhinol. 2016;6(21):45-8. doi: https://doi.org/10.1515/rjr$\underline{\text { 2016-0006 }}$ 